\title{
Temperature Controlled and Monitored Ex Vivo Lung Perfusion System for Research and Training Purposes
}

\begin{abstract}
Ex vivo lung perfusion (EVLP) is a preservation method for donor lungs, which keep lungs viable in a physiological environment outside of a body for a short period of time. EVLP is established clinically for lung transplantation. Experimental applications for EVLP are e.g. lung cancer research or medical device development and testing. For preservation, a lung is ventilated artificially in an organ chamber and perfused antegrade through the pulmonary artery. Here we introduce a thermoregulation system for an experimental EVLP system to be used for translational research approaches as well as for training medical staff. To implement physiological culture conditions that are a prerequisite for lung preservation and tissue homeostasis, a thermoregulation is needed to rewarm the explanted lung tissue (storage temperature $4^{\circ} \mathrm{C}$ ). Technically, the EVLP system must be thermally insulated, so loss of caloric is avoided. For monitoring, temperature sensors are integrated within the lung, in the organ chamber and in the afferent perfusate tube, whereby the measured values determine the thermoregulation. Initial tests using thermal packs (cooled to $4-6^{\circ} \mathrm{C}$ ) placed on a heating mat, as a part of the perfusion circuit, showed that the perfusate temperature falls to $34^{\circ} \mathrm{C}$, but restores after approximately 60 minutes $\left(36.5^{\circ} \mathrm{C}\right)$, whereby the thermal pack is warmed. With this setup longer perfusion times should be obtained rather than without thermoregulation due to normothermic perfusion of the lung.
\end{abstract}

Keywords: Ex vivo lung perfusion, ex vivo, lung perfusion, temperature control, temperature adjustment

https://doi.org/10.1515/cdbme-2019-0074

\footnotetext{
*Corresponding author: Christina Pongratz: INKA Institute of Medical Technology, Otto-von-Guericke-University (OVGU), Magdeburg, Germany, e-mail: christina.pongratz@ovgu.de Jens Ziegle, Axel Boese, Michael Friebe: INKA Institute of Medical Technology, OVGU, Magdeburg, Germany

Helena Linge, Thorsten Walles: Department of Thoracic Surgery, University Clinic for Cardiac and Thoracic Surgery, Otto-vonGuericke University, Magdeburg, Germany
}

\section{Introduction}

Lung transplantation has been established as the ultimate solution for patients with end-stage lung diseases. Due to a rising number of patients awaiting lung transplantation on one hand, and an increasing shortage of donor lungs on the other hand, about $50 \%$ of listed patients die while waiting for a transplantation [1-3]. Moreover, many donor lungs deteriorate following explantation and only $15-20 \%$ of donated lungs fulfill the functional requirements for lung transplantation $[2,4,5]$. To increase the number of transplantations, the quality of explanted donor lungs must be enhanced [2].

Ex vivo lung perfusion (EVLP) is a lung-preservation technique, where the donor lung is perfused and ventilated outside of the living body at body temperature for several hours. Commercially available EVLP systems mimic real physiologic conditions, for example, the provision of nutrients using a perfusion solution instead of blood, called perfusate, the body temperature by implementing a temperature adjustment and the ventilation of the lung using a ventilator. Using EVLP, a more accurate evaluation and eventually a reconditioning of potential donor lungs can be achieved $[1,4]$.

If pulmonary parameters are restored, it is possible to transplant this donor lung with a good outcome for the patient. Preclinical studies show that after a treatment using EVLP, both, normal and injured lungs, showed excellent lung function after transplantation. [4]

\section{State of the art}

There are four commercialized systems available on the market: the XPSTM system (XVIVO Perfusion, Göteborg, Sweden), LS1 (Vivoline Medical, Göteborg, Sweden), Lung Assist (Organ Assist, Groningen, Netherlands) and Organ Care System ${ }^{\mathrm{TM}}$ Lung (TransMedics, Andover, Massachusetts, U.S.A.) [6]. All systems are slightly different in their setup and in their concept of clinical use. The XPSTM is a device, which 
was designed for the requirements of the Toronto lung transplantation protocol. The LS1 (or LS) system from Vivoline Medical and the Lung Assist are other static systems. The most recent commercial system, the Organ Care System ${ }^{\mathrm{TM}}$ Lung, is, as opposed to the other three systems, a portable system. [6 - 10]

At the Magdeburg University, an EVLP system has been developed for translational research purposes and medical training. Specifications of this prototype are: 1 . Lean design to afford transportation, 2. easy-to-use, 3. cost-effectiveness by implementation of clinically established components and devices, and 4. open design to facilitate technical modifications and re-arrangements according to intended research application.

\section{Materials and methods}

The goal of this work, was to develop temperature monitoring and adjustment of the perfusate running through the explanted lung to mimic body temperature within the EVLP system, to improve biological function and outcome and increase perfusion times. The temperature of the organ should reach a temperature of $35^{\circ} \mathrm{C}$ with a range of $\pm 2^{\circ} \mathrm{C}$ with a permanent temperature of $36^{\circ} \mathrm{C}$ to $37^{\circ} \mathrm{C}$. A new setup of the system is necessary to prevent loss of caloric and to allow heating of the perfusate.

\subsection{Organ chamber}

For the development of temperature monitoring and adjustment, a new setup of the organ chamber is necessary, to minimize the loss of heat during perfusion. Besides the heat insulation of the organ chamber, appropriate accessories to interconnect subsystems to the organ chamber are needed. For a successful imaging of the perfused lung, the organ chamber must consist of a material with low X-ray absorption, otherwise the image quality would be limited. The proposed devices are not permitted to impair the imaging. The new developed organ chamber is shown in figure 1 .

\subsection{Perfusion circuit}

For the perfusion circuit a heating of the lung through the perfusate is used. Therefore, the perfusion circuit must be adjusted, so the perfusate can be slowly heated up to the intended temperature. The simplest solution is adding counter flow tubes to the current perfusion circuit. For that, a large tube carrying the heated water of the thermostat and a small tube, through which the perfusate flows are necessary. The small

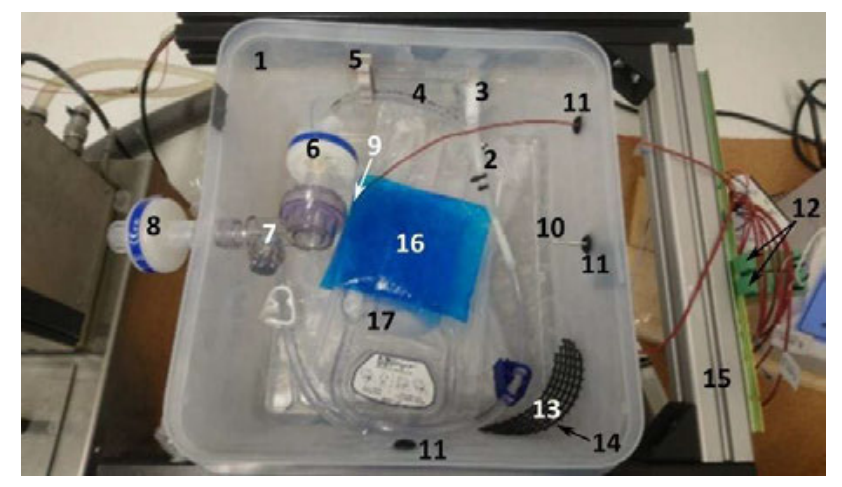

Figure 1: Test setup of the new organ chamber consisting of (1) a gastronorm box of GN size 2/3, (2) perfusion tube, connected (3) tube adaptor, (4) endotracheal tube, (5) clamp for the endotracheal tube, (6) heat and moisture exchanger, (7) patient valve, (8) bacteria filter, (9) Pt1000 sensor for leaking perfusate temperature, (10) Pt1000 sensor for air temperature in organ chamber, (11) cable sleeves, (12) transducers, (13) grid for avoiding an occlusion of the (14) effluent, (15) stillage of the organ chamber, (16) cool pack as substitute of a $4{ }^{\circ} \mathrm{C}$ cold lung that is wrapped around a (17) heating mat.

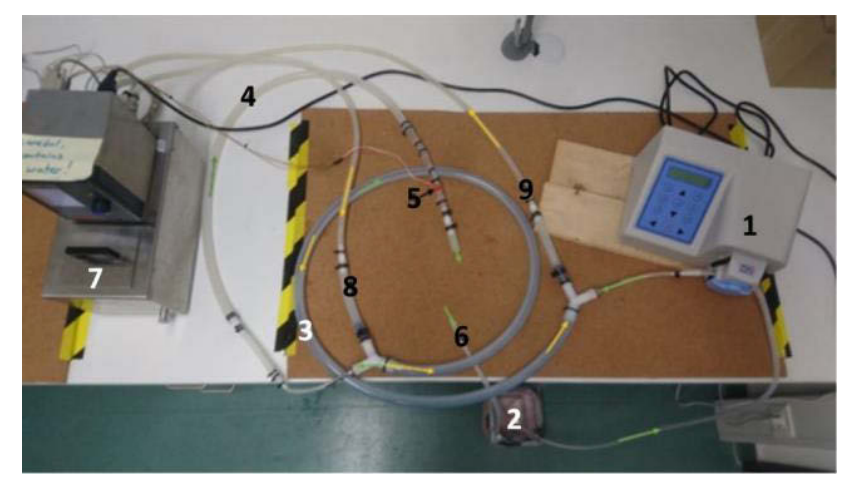

Figure 2: Overview about the experimental setup of the new perfusion circuit including the main parts for heating the perfusate. The perfusate circuit is labelled with green arrows, the heated water circuit with yellow ones. (1) Roller pump, (2) perfusate reservoir, (3) counter flow tubes, (4) afferent perfusate tube, (5) Pt100 sensor for adjusting the perfusate temperature, (6) efferent perfusate tube, (7) Huber CC-E thermostat, (8) efferent water tube from the Huber thermostat, (9) afferent water tube to the Huber thermostat

tube needs to have a very thin wall, so the amount of caloric transmitted through the wall is maximized. The new setup of the perfusion circuit is shown in figure 2 and for better visibility, the organ chamber, temperature sensors and stillage are removed. In the final setup, all parts of the circuit carrying perfusate are insulated to minimize the loss of caloric.

\subsection{Temperature monitoring and adjustment devices}

The new setup includes different temperature sensors, so the monitoring and adjustment can be achieved. There are three thermal sensors, one is integrated within the introductory perfusion tube. This is a Pt100 sensor connected to the Huber 
thermostat. The thermostat adjusts the perfusate temperature to the desired temperature. Another Pt1000 sensor is based within the lung, more precisely in the pulmonary vein. Both sensors, the Pt100 and the Pt1000, indicate how the temperature of the perfusate reduces whereas the lung increases its temperature due to heat transfer. The third sensor is placed within the organ chamber to observe the temperature within the organ chamber.

For an overview of all measured temperatures, a software tool was developed that reads all measured temperatures, shows them in graphic plots and writes the values into log files. The signals from the Pt1000 thermistors are add to transducers which finally read out with the software LabVIEW 2018 (National Instruments, Austin, Texas, U.S.A.) via the data acquisition device NI-DAQ USB-6009 (National Instruments, Austin, Texas, U.S.A.). In LabVIEW, the voltages are converted into temperatures using the linearity of Pt1000 and the correlation between resistance and temperature of the Pt1000. Finally, the temperatures and corresponding time stamps are saved into log files.

The third sensor, a Pt100 sensor, is directly plugged into the Huber thermostat that calculates the temperature internally. The measured external temperatures are integrated in LabVIEW by using a serial interface. After configuring the serial port, a request is sent to the thermostat. The response contains the desired external temperatures and are saved in the $\log$ file with the corresponding time stamp.

\subsection{Verification of the new test setup}

The new setup of the EVLP system was tested to verify the method of warming the porcine lungs using only the perfusate. To generate a suitable test environment, the setup was reduced on the main temperature regulatory parts, which include the organ chamber, the Huber thermostat, appropriate tubes, a pump and a perfusate reservoir. The measured values were shown in the running software on the computer.

A substitute for a cold porcine lung was used for the test setup consisting of an Irrigation Fluid Warming Set (Model 24750, 3M, Maplewood, Minnesota, U.S.A), called heating mat, and a cool pack $\left(8-10^{\circ} \mathrm{C}\right)$. The initial temperature of a porcine lung is usually about $8-10^{\circ} \mathrm{C}$ due to preparation arrangements at room temperature, which was the required starting point for the cool pack as well. The placement of the cool pack and heating mat is shown in figure 1 .

The heating mat was drained by the perfusate, which should warm the cool pack up to approximately $35^{\circ} \mathrm{C}$. During this test, the in-house compounded perfusion solution was substituted with water that had similar behavior within heating processes.
Before starting a test, the system was warmed, until a perfusate temperature of at least $35^{\circ} \mathrm{C}$ was measured (Pt100). Then, the cool pack was positioned within the organ chamber. This imitated the maximum warming of the cool pack as it is happening in the same way in a porcine lung.

\section{Results and discussion}

We performed three tests with one large cool pack with nearly the same results. As an example, one graph with measurements of temperatures is shown in figure 3 .

At the beginning of the measurements, the cool pack was wrapped around the heating mat. The perfusate temperature was at the beginning about $35.2^{\circ} \mathrm{C}$ and the air temperature measured $28^{\circ} \mathrm{C}$. The sensor, which measured the cool pack temperature noticed a large negative gradient of this temperature and displayed a temperature of about $21.7^{\circ} \mathrm{C}$. As the cool pack was placed within the organ chamber, the air temperature of the organ chamber decreased, due to a removal of the organ chamber lid, causing a loss of caloric towards the surroundings. The perfusate temperature decreased a few seconds later, because the cooled perfusate needed to pass the whole circuit until a temperature loss of the perfusate was noticeable. Sequentially, the cool pack was heated up through the heating mat and reached a temperature of nearly $34^{\circ} \mathrm{C}$. The air temperature increased continuously up to $30.1^{\circ} \mathrm{C}$.

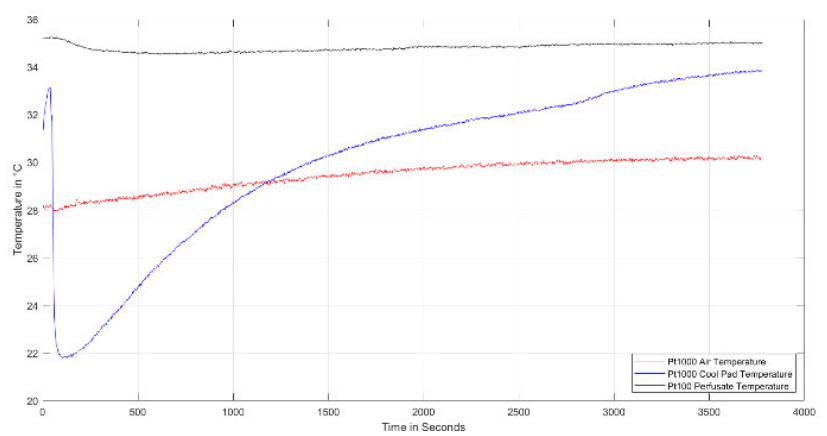

Figure 3: Measured air temperature (red), cool pack temperature (blue) and perfusate temperature (black) during one test using a cool pack and the test setup

Considering the three tests with cool packs, at all events it was possible to warm a porcine lung with the actual setup. As the porcine lungs weighed less than the large cool pack (lung: approximately $150 \mathrm{~g}$ depending on the weight of the pig vs. cool pack: $386 \mathrm{~g}$ ) and the porcine lungs have a smaller specific caloric capacity than the cooling liquid within the cool packs, it will need less time to warm the porcine lung up to $35^{\circ} \mathrm{C}$ to $37^{\circ} \mathrm{C}$ than during the tests with the cool packs. The new EVLP system setup is suitable for the warming cases, there is enough caloric transferred from the heated water from the thermostat to the perfusate. The lid of the organ chamber was useful to 
develop a constant environment regarding a constant temperature within the organ chamber. Additionally, the usage of the lid avoided an unnecessary loss of caloric during the tests.

\section{Conclusion}

The introduced temperature adjustment works well and a temperature adjustment of an EVLP device is possible. This will probably be achieved faster than the warming of the cool pack, due to a lower specific caloric coefficient, a lower weight, and a better perfusion of the lung tissue. For validating the development of edema during EVLP or an impact on the duration of EVLP by the temperature adjustment further tests with porcine lungs must be realized.

Regarding the accuracy of the sensors, corrections of the values might be necessary for a higher accuracy of the measured values. For now, the transducers of the Pt1000 sensors are calibrated at a temperature of $36^{\circ}$. One of the transducers could be calibrated to $30^{\circ} \mathrm{C}$, as this sensor measures the air temperature within the organ chamber. Otherwise, other transducers could be used.

The Pt100 sensor, that adjusts the temperature of the perfusate, has a high deviation from the real actual value. This is caused by the variations of this sensor. It is necessary to use a Pt100 sensor, as it is the only supported type of sensor by the Huber thermostat. It needs to have a diameter of maximum 2 $\mathrm{mm}$, so the sleeve for this sensor for protecting it from water is fitting. The used sensor has initially a cable length of only $30 \mathrm{~cm}$. This was lengthened, probably causing a change in the overall resistance of the Pt100. These adaptions lead to a nonlinear temperature offset, so a simple correction of it could not be achieved that easy.

The actual implemented electronics are fixed at the stillage of the organ chamber. Using a housing with at least protection category IP 55, a protection against dust or water could be implemented.

As the new setup shall be used in translational research, there have to be some changes in the process of EVLP. Regarding the intended Toronto protocol, the warming of the lung needs to take place before the ventilation is initiated [11]. The perfusion rate should be set to 5 or 6 milliliters per minute to heat the perfusate as much as possible. After initiating the ventilation, it is also possible to change the perfusate flow rate, so the experiment can take place under fixed circumstances.

For further development, more sensors, e.g. pH-sensors, perfusate pressure sensors or humidity sensors within the organ chamber, could be included. Further tests can be realized to prove, whether the implemented temperature adjustment is suitable for the heating of a porcine lung, to investigate, whether the temperature adjustment has an impact on the outcome of the actual EVLP results and whether angiography can be proceeded with this setup.

\section{Author Statement}

Research funding: The author state no funding involved. Conflict of interest: Authors state no conflict of interest. Informed consent: Informed consent was not required for this study. Ethical approval: Ethical approval was not required for this study.

\section{References}

[1] A. Koch, N. Pizanis, C. Olbertz, O. Abou-Issa, A. Slama, C. Taube, C. Aigner, H. Jakob, and M. Kamler. Ex-vivoLungenperfusion: Werkzeug zur optimierten Nutzung von Spenderorganen. Zeitschrift für Herz-,Thorax- und Gefäßchirurgie; 2017;32.

[2] K. Nelson, C. Bobba, S. Ghadiali, D. Hayes, S. Black, and B. Whitson. Animal models of ex vivo lung perfusion as a platform for transplantation research. World Journal of Experimental Medicine; 2014.

[3] L. Munshi, S. Keshavjee, and M. Cypel. Animal models of ex vivo lung perfusion as a platform for transplantation research. The Lancet Respiratory Medicine; 2013.

[4] M. Cypel, J. Yeung, M. Liu, M. Anraku, F. Chen, W. Karolak, M. Sato, J. Laratta, S. Azad, M. Madonik, C.-W. Chow, C. Chaparro, M. Hutcheon, L. G. Singer, A. S. Slutsky, K. Yasufuku, M. de Perrot, A. F. Pierre, T. K. Waddell, and S. Keshavjee. Normothermic ex vivo lung perfusion in clinical lung transplantation. New England Journal of Medicine; 2011;364(15):1431-1440. PMID: 21488765.

[5] G. Makdisi, T. Makdisi, T. Jarmi, and C. C. Caldeira. Ex vivo lung perfusion review of a revolutionary technology. Annals of translational medicine; 2017.

[6] D. Van Raemdonck, A. Neyrinck, M. Cypel, and S. Keshavjee. Ex-vivo lung perfusion. Transplant International; 2015;28(6):643-656.

[7] XVIVO Perfusion. Xps ${ }^{\mathrm{TM}}$ The flexible comprehensive EVLP platform; 2019.

https://www.xvivoperfusion.com/products/xps/, Last access: 18/04/2019.

[8] Organ Assist. Lung assist; 2019. https://www.organassist.nl/products/lung-assist, Last access: 18/04/2019.

[9] TransMedics. Ocs ${ }^{\mathrm{TM}}$ Lung; 2017. http://www.transmedics.com/wt/redirect/lung_preservation_m ed.html, Last access: 20/03/2019.

[10] XVIVO Perfusion. Xvivo Is; 2016. https://www.xvivoperfusion.com/products/ls/, Last access: 18/04/2019.

[11] M. Cypel, J. C. Yeung, T. Machuca, M. Chen, L. G. Singer, K. Yasufuku, M. De Perrot, A. Pierre, T. K. Waddell, and S. Keshavjee. Experience with the first 50 ex vivo lung perfusions in clinical transplantation. The Journal of Thoracic and Cardiovascular Surgery; 2012;144(5):1200 -1207. 